\title{
Viruses without borders
}

\author{
Lindsay E Nicolle MD FRCPC, Editor-in-chief, University of Manitoba, Winnipeg, Manitoba
}

$\mathrm{I}_{\mathrm{n}}^{\mathrm{n}}$ nfluenza is finally getting the respect that it deserves. Over the past several years, the scientific, public health and general public profile of this illness has been heightened by several, largely independent, developments.

The decrease in number of acute care beds across Canada over the past decade, coupled with an aging population and our extraordinary success in extending the survival of patients with significant chronic illness, has eliminated any cushion in bed occupancy in the hospital system. The flexibility in bed availability to accommodate the predictable seasonal variation in bed demand is gone. Increased illness requiring hospitalization in the winter respiratory season, a large part of which is due to influenza, now pushes the acute health care system beyond capacity. In effect, influenza is a sentinel virus for acute health care capacity. The result is that, on a yearly basis, the press trumpets the Armageddon of emergency room collapse and medical bed deficits, and influenza is identified as the cause.

This past winter, Canada-wide influenza activity maps, continually updated, appeared on the weather channel and in the pages of The Medical Post. The arrival of the neuraminidase inhibitors for influenza treatment (1) and the substantial pharmaceutical funding now available for promotion of these drugs created these 'flu maps. The limited efficacy of these new agents for the treatment of influenza can be regretted, and we can hope that therapeutic use will not interfere with a prophylactic role, where the drugs are likely to have a large impact. Although clinical trials and in vitro observations do not suggest resistance emergence is a major problem, widespread use in infected populations will certainly promote resistance. So the marketing of neuraminidase inhibitors also raises the influenza profile and, in fact, promotes a major change in practice by spreading the message that individuals who think that they have the 'flu should see their physician.

The third item highlighting influenza received popular press several years ago, and continues to haunt infectious disease epidemiologists and public health officials. This, of course, is the outbreak of "chicken 'flu" in Hong Kong over the Christmas season of 1997 (2). An entirely new influenza virus, an H5N1 strain, not previously isolated in infected humans, emerged. The strain was acquired by exposure to infected chickens and did not transmit readily between humans. Infected individuals were young, with a mortality rate of $30 \%$. The success of the influenza virus as a pathogen is, of course, largely due to its promiscuity in genetic reassortment (3). A reasonable assessment of the Hong Kong outbreak is that we were one genetic reassortment, allowing human-to-human transmission, from pandemic catastrophe. Fortunately, the aggressive, 'no half measures' approach of complete destruction of the chicken flocks of Hong Kong successfully limited this outbreak.

Influenza outbreaks occur yearly in the winter in temperate climates. Because the virus continually modifies its antigenic structure, long term immunity following infection is elusive. Periodically, previously thought to occur every 10 years, pandemic influenza arises with the introduction of a substantially genetically altered strain that spreads rapidly through the nonimmune world population (2). We are overdue for the next pandemic. The chicken 'flu outbreak in Hong Kong has us looking back to the 1918 influenza pandemic, which caused tens of millions of deaths worldwide, played a major role in ending the first World War, and was characterized by high rates of illness and very high mortality in healthy young adults. Is this the future?

Our understanding of what happened in 1918, and why, is incomplete, although expanding through current genetic technology. This virus emerged in the United States (Kansas, in fact) and was transported to Europe with troop movements of World War I. Thus, it is a variation of the observation that new influenza strains emerge from Asia. The extraordinary mortality in young, healthy individuals in the 1918 pandemic has not been adequately explained but is thought to represent unique virulence characteristics of that strain (4). New information elicited from DNA fragments retrieved from Alaskans who died in this epidemic and who were buried in permafrost may answer some of the remaining questions.

Influenza primarily causes mortality through secondary bacterial infections in elderly individuals with comorbidities. However, primary influenza pneumonia is a well recognized, clinical presentation, which kills even young healthy people rapidly. We do not yet know whether new therapies will be effective in the treatment of the more severe manifestations at- 
tributable to influenza. The public health response will differ depending on the clinical and epidemiological features of the particular influenza strain. Recent experience with yearly outbreaks has focused on excess morbidity and mortality in the elderly, and future outbreaks will continue to have greatest impact in the elderly (5). The management of pandemic disease will require, then, a large scale intensification of current approaches of vaccinating target groups, prophylaxis in long term care facilities and other selected populations, and provision of sufficient acute care services. If, however, a strain similar to that of 1918 emerges, ie, illness and substantial mortality in young healthy individuals, a pandemic could de-stabilize our society. When pandemic influenza next arrives, we can also expect more rapid transmission than in the past because of increased global travel. Pandemic influenza is unique compared with other infectious or noninfectious catastrophes, including bioterrorism, because it will occur simultaneously across this country. Other jurisdictions will not be able to provide assistance. The response of the military and other public bodies, key responders in recent catastrophes in Canada, will also be limited because of the impact of the illness on these groups.

Fortunately, our public health providers at global (6), federal and provincial levels have heeded the warning, and identified planning for pandemic influenza as a priority. In Canada, the Bureau of Infectious Diseases at the Laboratory Centre for Disease Control (LCDC) has been a leader in this process. An initial priority has been ensuring a sufficient vaccine supply for Canada when pandemic influenza occurs. LCDC is also leading a collaborative planning process with the provinces, in communication with the World Health Organization, to develop a Canadian Contingency Plan for Pandemic Influenza. This plan includes ensuring vaccine supply and distribution, a surge capacity in health care delivery services, use of antivirals for treatment and prophylaxis, and laboratory and communications recommendations. A consensus document is being developed following a meeting, with wide government and professional representation, held in January 2000, hosted by LCDC. This document will provide the framework for federal and provincial planning.

While the public health agenda is reassuring, the prospect of pandemic influenza remains sobering. In the worst case scenario, the prospect is terrifying. Even with excellent planning, sufficient vaccine availability and optimal use of our current antivirals, catastrophe may not be avoided, especially with rapid importation into the country following initial identification. The magnitude and complexity of the issue suggests that the only truly successful response is through improved technology. Specifically, improvements in influenza vaccination production and effectiveness, and continued development and evaluation of antivirals are likely to have the greatest impact.

We are overdue for pandemic influenza. The chicken 'flu outbreak in Hong Kong was a wake-up call, and our public health leaders are responding appropriately. Aggressive management of yearly influenza outbreaks will give experience, and develop an infrastructure, which may be a foundation for a pandemic response. The current heightened public and scientific profile of influenza will help in achieving resources for planning. Pandemic influenza will arrive, and we need to be able to respond.

\section{REFERENCES}

1. Neuraminidase inhibitors for treatment of influenza A and B infections. MMWR Morb Mortal Wkly Rep 1999;48(RR-14):1-9.

2. Snacken R, Kendal AP, Haaheim LR, Wood JM. The next influenza pandemic: Lessons from Hong Kong, 1997. Emerging Infect Dis 1999;5:195-202.

3. Webster RG. Influenza: An emerging disease. Emerging Infect Dis 1998;4:436-41.

4. Glezen WP. Emerging infections: Pandemic influenza. Epidemiol Rev 1996;18:64-76.

5. Prevention and control of influenza: recommendations of the Advisory Committee on Immunization Practices (ACIP). MMWR Morb Mortal Wkly Rep 1999;48(RR-4):1-28.

6. World Health Organization. Influenza Pandemic Preparedness Plan. The Role of WHO and Guidelines for National and Regional Planning (WHO/CDS/CSR/EDC/99.1). Geneva: World Health Organization, 1999. 


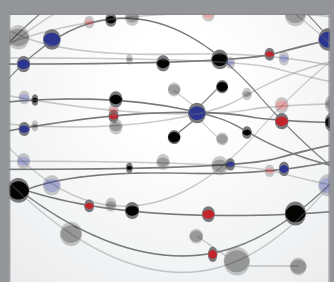

The Scientific World Journal
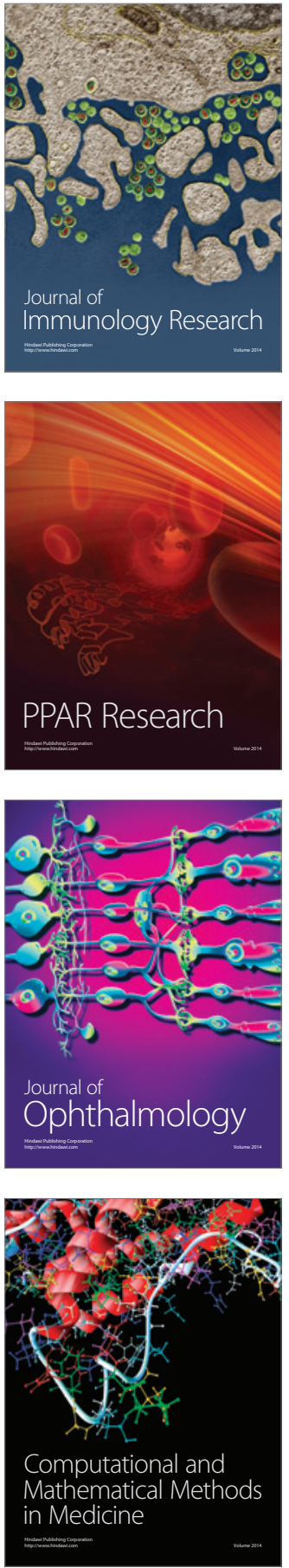

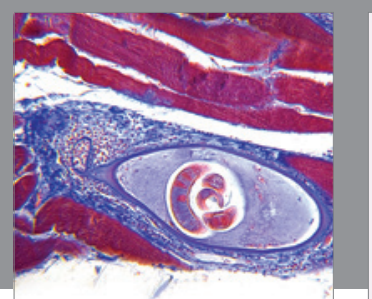

Gastroenterology Research and Practice

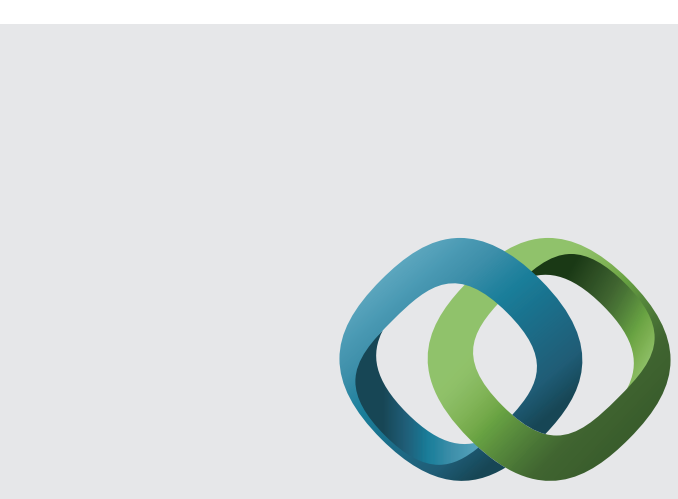

\section{Hindawi}

Submit your manuscripts at

http://www.hindawi.com
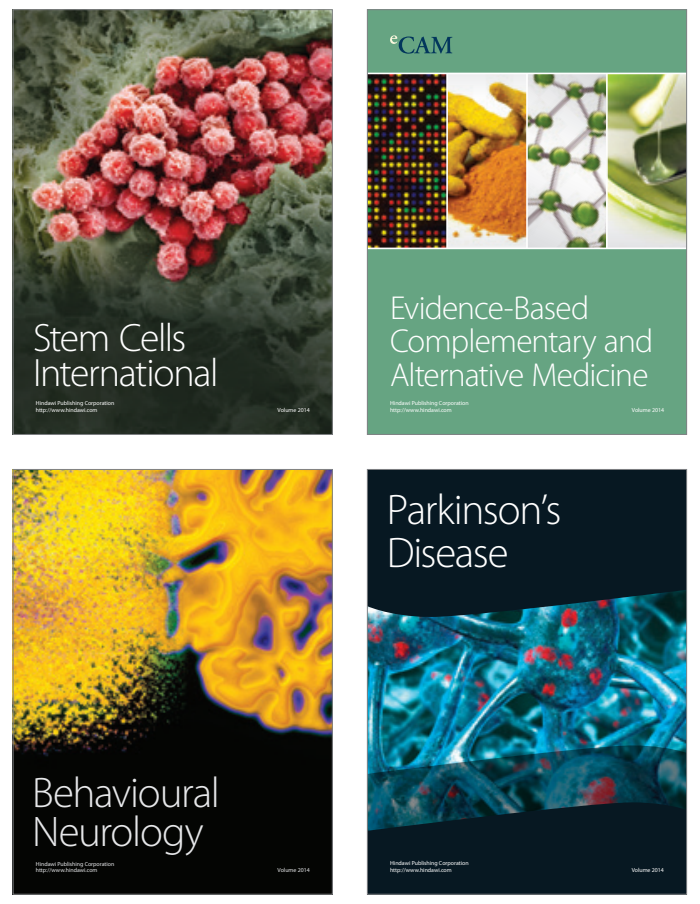
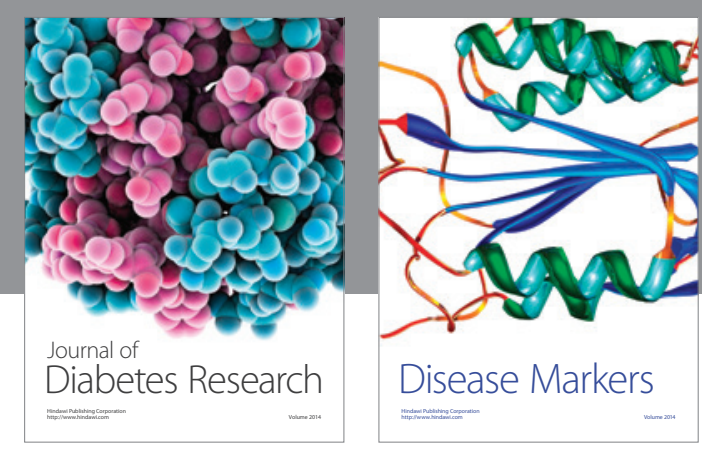

Disease Markers
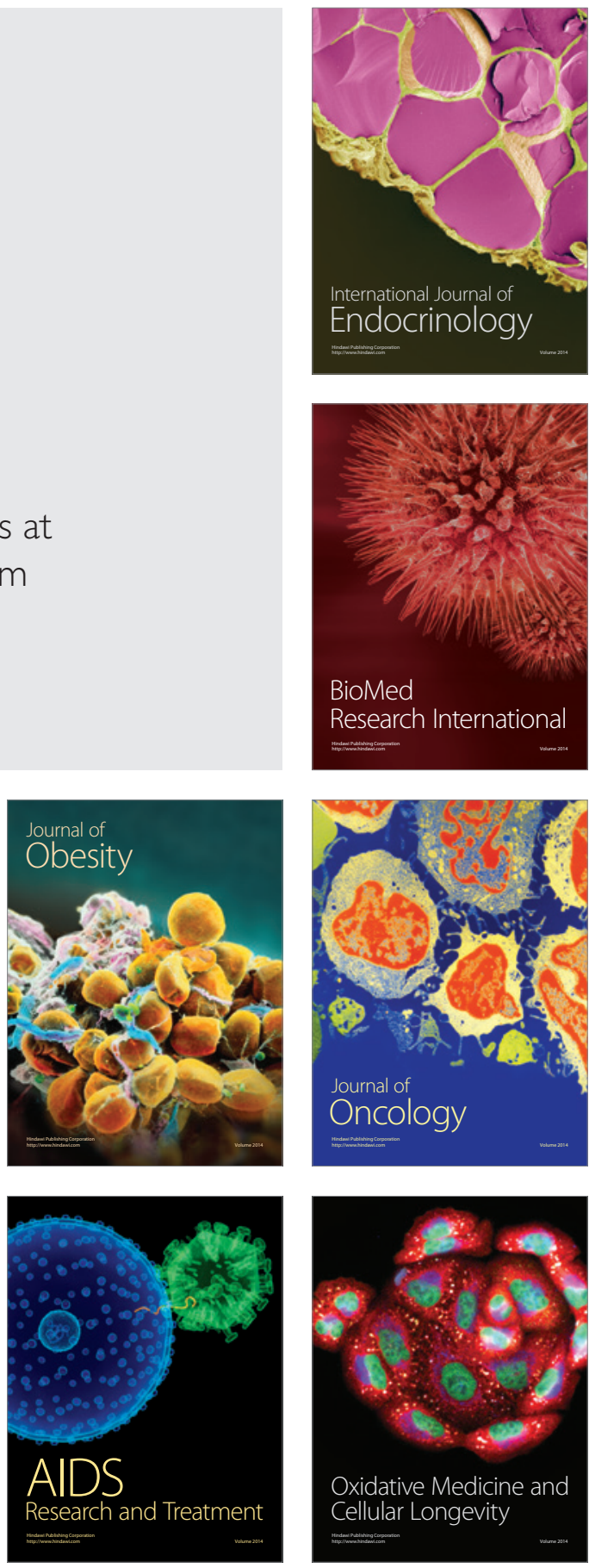